\title{
PRODUCTION BIOLOGIQUE DES SUBSTANCES CONCOURANT A LA FORMATION DE L'AROME DU BEURRE
}

\author{
par \\ JEAN PIEN \\ Ingénieur ehimiste, Docteur ès Sciences \\ Directeur des Laboratoires de la S.A.F.R. \\ (fin)

\section{DEUXIËME PARTIE}

\section{Biochimie de la formation de l'arome}

On ne connaît pas encore avec certitude le mécanisme de la formation de l'acétylméthylcarbinol et du diacétyle dans les cultures de ferments producteurs d'arome.

L'incertitude est d'autant plus grande que, d'une part, des germes très différents sont capables de produire ces substances, d'autre part, dans certains cas, la présence de l'acide citrique est indispensable et, dans d'autres cas, la formation d'acétoïne se produit dans les milieux sucrés n'en renfermant pas.

On peut donc supposer que l'on a affaire à plusieurs mécanismes différents dont les deux principaux seraient les suivants :

1. Formation d'acétoīne à partir de l'acide citrique (cas des bétacoques).

2. Formation d'acétoïne à partir du lactose (cas des strep. lactiques).

Quelle que soit l'hypothèse à laquelle on s'arrête il ne faut pas oublier que, même en milieu citrique, la présence des sucres est nécessaire. Ils disparaissent plus ou moins pendant la fermentation. Il est légitime de penser qu'ils y prennent part et que, au moins en partie, il y a là des étapes communes avec les fermentations déjà connues du glucose.

Or on sait que les fermentations des hexoses ont un point de départ commun et une longue étape initiale commune :

1. Passage de la forme glucopyranose à la forme glucofuranose (sous l'influence de l'hexokinase de Meyerhof). La forme furanique obtenue est très peu stable.

2. Phosphorylation du glucose sous l'influence de la cozymase (acide adénosine triphosphorique). Il se forme des hexoses mono et di-phosphoriques (esters de Robison, de Harden et Young, de Neuberg...). Cette phosphorylation est tout à fait générale.

3. Rupture de l'aeide hexose-diphosphorique en deux molé- 
cules d'acides triosephosphoriques (acide dioxycétone phosphorique) sous l'influence de la zymohexase.

4. Dismutation des deux molécules de trioses (l'une est réduite en acide glycérophosphorique, l'autre est oxydée en acide phosphoglycérique).

5. Décomposition de l'acide phosphoglycérique en acide phosphorique et acide pyruvique.

Jusque là, le mécanisme est commun à toutes les fermentations du glucose. C'est à partir de ce point que l'on observe des divergences :

Dans un groupe de fermentations, l'acide pyruvique est décarboxylé par la carboxylase et donne naissance à l'acétaldéhyde et au gaz carbonique. alors :

A l'intérieur de ce groupe, de nouvelles divergences apparaissent

Dans la fermentation alcoolique, par exemple, l'acétaldéhyde, jouant le rôle d'accepteur d'hydrogène, oxyde le glucose en acide phosphoglycérique (en présence de phosphates) tandis qu'ellemême est réduite en alcool éthylique.

Dans d'autres fermentations l'aldéhyde se condense en aldol (aldolisation) lequel subit des oxydations, des réductions, des hydrolyses qui permettent d'expliquer la génèse d'un grand nombre de produits (acide butyrique, alcool butylique, etc.).

Or l'acétoïne, le diacétyle, le butylèneglycol sont aussi des corps en $\mathrm{C}^{4}$. Il est difficile de concevoir que l'on pourra les former, s'ils prennent vraiment leur origine dans les sucres, sans une condensation des divers corps en $\mathrm{C}^{3}$ résultant du clivage des glucides. C'est pourquoi on est conduit à penser aux passages suecessifs suivants :

Glucose (issu du lactose éventuellement) $\rightarrow$ acide pyruvique $\rightarrow$ acétaldéhyde $\rightarrow$ aldol.

Que se passe-t-il alors si l'on attribue aux substances en $\mathrm{C}^{4}$ une origine citrique? S'agit-il d'un mécanisme différent traduisant des origines différentes ou, au contraire, peut-on rapporter aux sucres l'origine de l'acétoïne?

$$
*^{*} \text { * }
$$

\section{Formation d'acétoïne à partir de l'acide citrique. Schéma de Van Beynum (cas des bétacoques)}

Pour VAN Beynum, le lien étroit constaté expérimentalement (dans le cas des bétacoques : Str. citrovorus) entre l'apparition de l'acétoïne et la disparition de l'acide citrique permet de penser 
que ce dernier est la source directe à partir de laquelle l'acétylméthylcarbinol prend naissance.

Comment peut-on concevoir le passage de l'acide citrique à l'acétoïne? Les stades intermédiaires acide pyruvique et acétaldéhyde peuvent-ils être envisagés ?

Voici, brièvement résumé, le schéma de VAN BEYnum [12] :

1. Une molécule d'acide citrique serait tout d'abord scindée en une molécule d'acide acétique, une molécule de gaz carbonique et une molécule d'acide pyruvique :

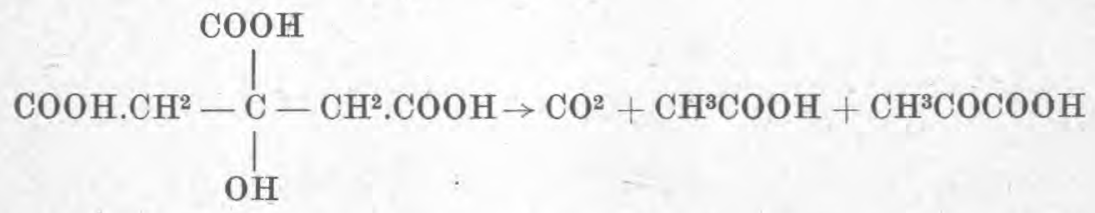

2. L'acide pyruvique serait ensuite décarboxylé (comme il l'est dans plusieurs fermentations des glucides) et donnerait une molécule de $\mathrm{CO}^{2}$ et une molécule d'acétaldéhyde sous l'influence d'une carboxylase :

$\mathrm{CH}^{3} \mathrm{COCOOH} \rightarrow \mathrm{CO}^{2}+\mathrm{CH}^{3} \mathrm{CHO}$

3. L'acétaldéhyde formée réagirait alors de deux manières :

a) En milieu acide elle se condenserait sous l'influence d'une carboligase pour donner une molécule d'aldol :

\section{$\mathrm{CH}^{3} \mathrm{CHO}+\mathrm{CH}^{3} \mathrm{CHO} \rightarrow \mathrm{CH}^{3} \mathrm{CHOH} . \mathrm{CH}^{2} \mathrm{CHO}$}

qui s'isomériserait en acétylméthylcarbinol $\mathrm{CH}^{3} \mathrm{CHOHCOCH}{ }^{3}$ ou encore, la condensation de l'aldéhyde donnerait directement l'acétoïne.

$\mathrm{CH}^{3} \mathrm{CHO}+\mathrm{HOC} . \mathrm{CH}^{3} \rightarrow \mathrm{CH}^{3} \mathrm{CHOH} . \mathrm{COCH}^{3}$

exactement comme il se forme du phénylacétylcarbinol quand on introduit de l'aldéhyde benzoïque dans une fermentation alcoolique

$\mathrm{C}^{6} \mathrm{H}^{5} \mathrm{CHO}+\mathrm{HOC} . \mathrm{CH}^{3} \rightarrow \mathrm{C}^{6} \mathrm{H}^{5}$. CHOH.CO. $\mathrm{CH}^{3}$

b) En milieu neutre l'acétaldéhyde subirait une réaction de Cannizzaro : une molécule s'oxyde en acide acétique, une autre est réduite simultanément en alcool éthylique.

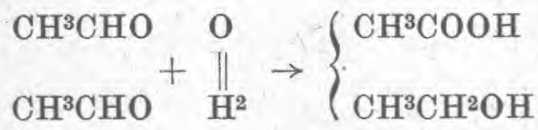

Il convient de noter que, en milieu neutre, seule la réaction $b$ ) intervient, alors qu'en milieu acide les deux peuvent se produire. Ce qui revient à dire que : 
En milieu neutre il se forme seulement de l'acide acétique (et de l'alcool).

En milieu acide-il se forme de l'acétoïne, de l'acide acétique (et de l'alcool).

Le bilan total de la fermentation serait done le suivant :

Une molécule d'acide citrique donne naissance à :

En milieu neutre :

2 mol. de $\mathrm{CO}^{2}+1,5 \mathrm{~mol}$. d'acide acétique $+1 / 2 \mathrm{~mol}$. d'alcool éthylique.

En milieu acide :

$2 \mathrm{~mol} \cdot \mathrm{CO}^{2}+\mathrm{x}$ mol. d'acétoïne $+(1,5-\mathrm{x})$ mol. d'acide acétique $+(0,5-\mathrm{x}) \mathrm{mol}$. d'alcool.

La valeur maximum de $\mathrm{x}$ étant $1 / 2$ molécule d'acétoïne.

Cas limite (en milieu acide) :

2 mol. $\mathrm{CO}^{2}+0,5$ d'acétoïne +1 acide acétique (sans alcool).

Sehéma de Van Beynum:

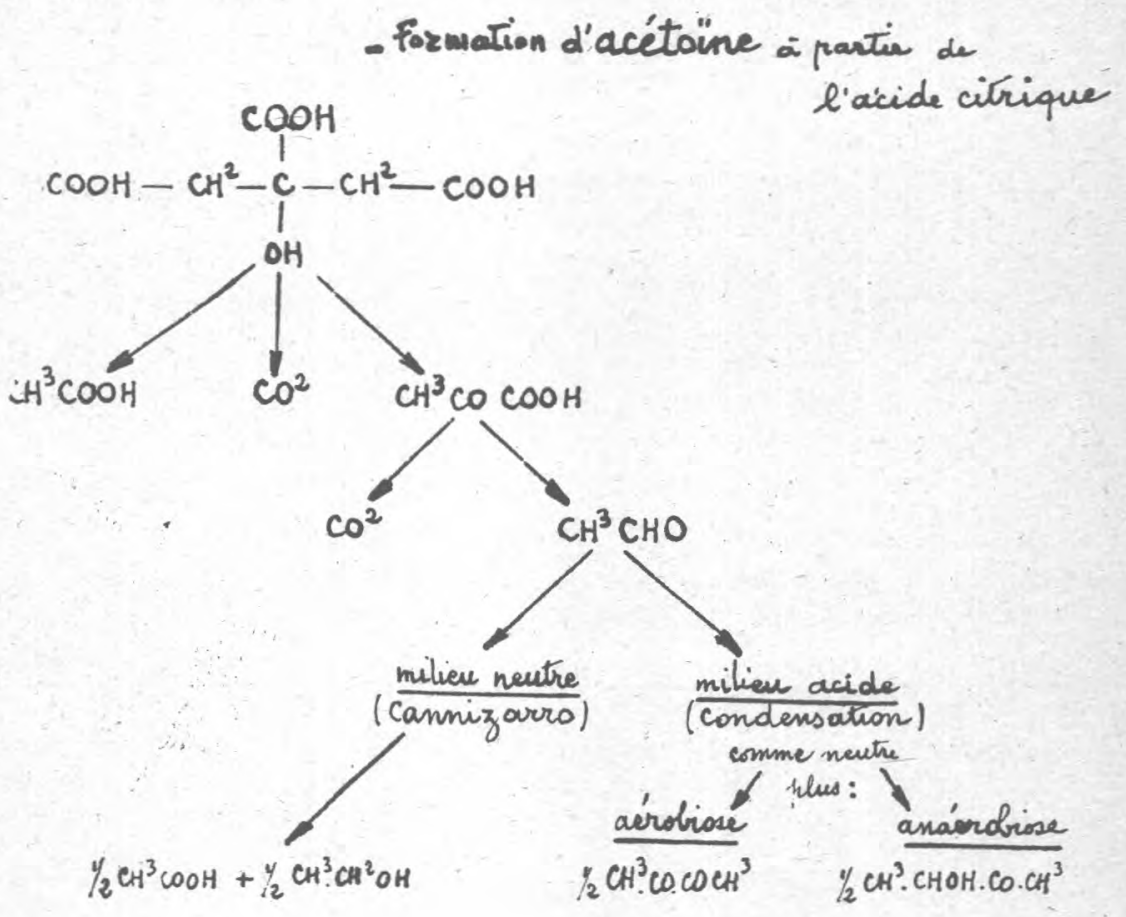


Selon VAN Beynum le dosage des produits de la fermentation confirme cette théorie.

Ce schéma rend compte de tous les phénomènes observés dans les cultures de bétacoques d'arome.

\section{Formation d'acétoïne à partir du lactose . (cas des Str. lactiques aromatiques)}

Il est avéré que les ferments d'arome qui n'appartiennent pas au groupe des bétacoques (en particulier les variétés aromatiques de Str. lactis) produisent de l'acétoïne sans décomposer l'acide citrique puisque le taux de celui-ci (quand il est présent dans les cultures) ne varie pas.

On peut, semble-t-il, faire à ce sujet l'hypothèse suivante :

1. Hydrolyse du lactose (en glucose et galactose) par la lactase de ces germes qui sont des lactiques vrais et acidifient fortement le milieu par eux-mêmes.

2. Transformation du glucose et du galactose en acide pyruvique (en suivant le processus classique avec passage intermédiaire par les trioses et leurs dérivés de dismutation).

3. Décarboxylation de l'acide pyruvique (au moins en partie) en acétaldéhyde, condensation ou cannizarisation suivant le $p H d u$ milieu, etc., comme dans le schéma de Van Beynum.

Entre les deux schémas, la différence porte sur l'origine de l'acide pyruvique : à partir de l'acide citrique dans le premier cas, à partir du lactose dans le second.

\section{Remarque :}

Les ferments d'arome qui n'attaquent pas l'acide eitrique sont des producteurs d'acide lactique au même titre que les Str. lactiques vrais (dont ils ne sont qu'une variété).

Donc, à côté de la formation d'acétoïne, il faut expliquer la formation de l'acide lactique (qui tire évidemment son origine du lactose).

Or dans les théories de Embden, Meyerhof..., l'acide lactique peut être formé par réduction de 1'acide pyruvique qui, réagissant sur l'acide glycérophosphorique, donne par dismutation entre les deux molécules : l'acide lactique (réduction de l'acide pyruvique), et régénération de l'acide triosephosphorique (oxydation de l'acide glycérophosphorique). 
Acide glycérophosphorique
$\mathrm{CH}^{2} \cdot \mathrm{O} \cdot \mathrm{PO}(\mathrm{OH})^{2}$<smiles>CC(C)O</smiles>

।
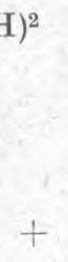

$\mathrm{COOH}$

Acide pyruvique<smiles>C1CCCC1</smiles>

$\mathrm{CH}^{3}$
$\mathrm{CH}^{2}, \mathrm{O} \cdot \mathrm{PO}(\mathrm{OH})^{2}$<smiles>C1CCCC1</smiles>

O $\mathrm{CH}^{2} \mathrm{OH}$

$\mathrm{H}^{2}$

$\mathrm{COOH}$ (acide

triosephosphorique

régénéré qui rentre dans le cycle)

$\mathrm{CHOH}$

(acide lactique)

On aurait donc, parallèlement :

Transformation d'une partie de l'acide pyruvique en acide lactique (suivant le schéma ci-dessus).

Transformation d'une autre partie de l'acide pyruvique en acétoïne (suivant le schéma de Van Beynum).

Le schéma d'ensemble ci-dessous rend compte des deux séries de phénomènes :

Formation dacétoine à partir dus Sucres en l'absence d'ecide citrique avec fermentat. Lactique associée. - (Cas Les Sti. Lecterque aromatiquas)

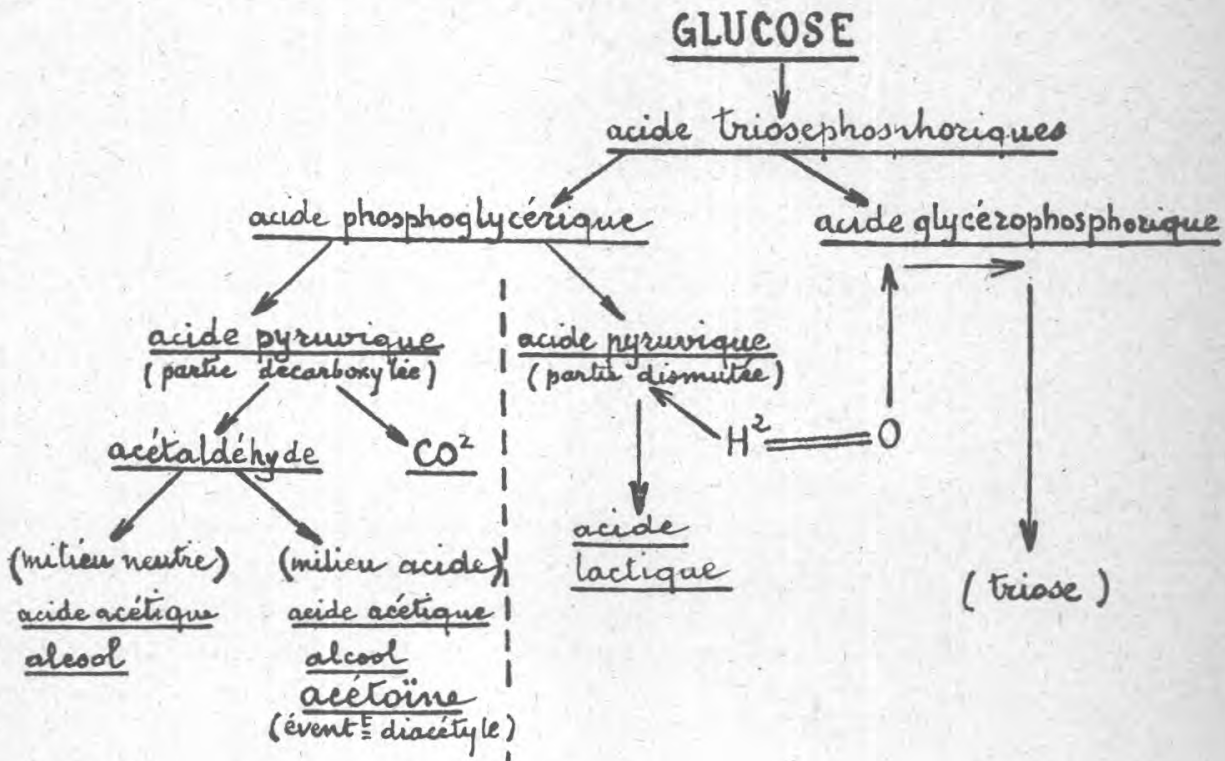

Fin du Sohima de Vase Beyneem I 


\section{Critique du schéma de Van Beynum. Idée d'un schéma nouveau (cas des bétacoques)}

Si la disparition de l'acide citrique dans les cultures de bétacoques n'est pas douteuse, il est permis néanmoins de se demánder si cet acide est bien la cause directe de la formation d'acétoïne ou si ce ne sont pas les sucres, comme dans le cas des str. lactiques producteurs d'arome, qui seraient à l'origine de l'acétoïne (l'acide citrique jouant, par sa disparition, un rôle nécessaire, mais n'étant pas par lui-même la substance mère). En d'autres termes, est-il possible de relier la formation d'acétoïne à une source unique qui serait le lactose (glucose) dans tous les cas ?

VIRTANen et ses Collaborateurs [13] cherchant à rattacher la formation de l'acétoïne à la fermentation du glucose ont pu montrer que :

1. Les bétacoques d'arome ne forment pas d'acétoïne à partir du glucose seul.

2. Ils en donnent si la solution glucosée est additionnée d'un accepteur d'hydrogène (bleu de méthylène, quinone...).

3. Les bactéries d'arome ne forment pas d'acétoïne à partir de l'acide citrique seul, ni en présence d'un accepteur d'hydrogène.

4. Elles en donnent à partir de solutions contenant du glucose et de l'acide eitrique.

Dans ces conditions, conclut VIRTANEN, les-substances aromatiques se forment à partir du sucre et non à partir de l'acide citrique. La formation à partir du sucre exige la mise en ouvre d'un accepteur d'hydrogène convenable qui peut être précisément l'acide citrique ou ses produits de décomposition dont le rôle dans ces conditions serait toujours nécessaire, mais différent de celui que lui attribue VAN BEYNUM.

Ces données importantes ont été récemment confirmées par les recherches encore inédites de GiLLIs [32].

Essayons de dégager le mécanisme fermentaire qui résulterait de ce rôle particulier de l'acide citrique.

Dans la fermentation alcoolique du sucre il y a, avons-nous dit, formation d'acétladéhyde qui oxyde le glucose en jouant le rôle d'accepteur d'hydrogène, ce qui a pour résultat de réduire l'aldéhyde en alcool.

Dans la fermentation qui conduit à l'acétoïne il y aurait oxydation d'un acide triosephosphorique dérivé du glucose sous l'influence d'un accepteur d'hydrogène qui serait cette fois l'acide citrique (1), lequel serait réduit en acétoïne comme nous allons le voir.

(1) Ou l'un de ses produits de décomposition. 
D'autre part la décarboxylation de l'acide pyruvique (valable dans les deux hypothèses) ou même d'un $\mathrm{COOH}$ de l'acide citrique (hypothèse de VAN BEYNUM) reste nécessaire. Rien ne s'oppose done à ce qu'on envisage la décarboxylation de $2 \mathrm{COOH}$ de l'acide citrique.

On aurait done :

1. Décarboxylation de l'acide citrique :<smiles>CC(O)(O)C(C(=O)O)C(=O)O</smiles>

2. Réduction simultanée de ce corps jouant le rôle d'accepteur d'hydrogène dans la fermentation du glucose :<smiles>CC(O)CC=CC(O)C=O</smiles>

Le corps formé n'est autre que $\mathrm{CH}^{3} \mathrm{CHOH} . \mathrm{COCH}^{3}$, e'est-à-dire l'acétoïne.

Il est intéressant de noter que le corps résultant de la décarboxylation de l'acide citrique n'est autre que le dérivé hydroxylé (en $\alpha$ ) de l'acide isobutyrique.

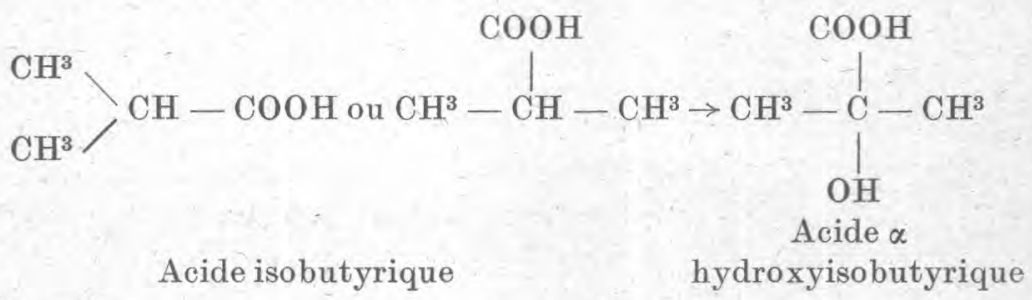

Or HAMMER et ses collaborateurs [33] ont précisément montré, en étudiant la formation d'acétoïne à partir d'autres produits que l'acide citrique, que l'acide $\alpha$ hydroxyisobutyrique en fournissait aisément.

\section{$\mathrm{COOH}$}

L'acide tricarballylique $\mathrm{COOH} . \mathrm{CH}^{2}-\mathrm{CH}-\mathrm{CH}^{2} \mathrm{COOH}$ qui ne diffère du précédent (aux $\mathrm{COOH}$ près) que par l'absence du groupement $O H$, ne conduit pas à l'acétoïne. L'acide isobutyrique n'en donne pas non plus. Ce sont là des preuves que ce groupement $\mathrm{OH}$ 
est indispensable et que l'acide $\alpha$ hydroxyisobutyrique est bien le chemin possible de passage de l'acide citrique décarboxylé, à l'acétoïne.

De même la fermentation de l'acide $\alpha$ hydroxyisobutyrique ne donne pas d'acides volatils. Done le $\mathrm{COOH}$ de la formule n'est pas détaché et est impliqué dans la formation de l'acétoïne - ce qui renforce encore notre hypothèse dans laquelle ce $\mathrm{COOH}$ est réduit pour donner le groupement eétonique de l'acétoïne.

Le travail de HAMMER constitue une confirmation expérimentale de notre hypothèse. On peut donc admettre que l'acide citrique, jouant le rôle d'accepteur d'hydrogène, est, après décarboxylation en acide $\alpha$ hydroxyisobutyrique, réduit en acétoïne.

3. Pour compléter ce schéma il convient de dire que le glucose (ou ses trioses de décomposition) ainsi oxydé par l'acide $\alpha$ hydroxyisobutyrique, suit le chemin habituel de la fermentation des sucres. Il y a lieu de remarquer toutefois que l'oxydation des acides triosephosphoriques en acide phosphoglycérique rend impossible la dismutation des trioses entre eux et ne permet pas l'apparition d'acide glycérophosphorique. De ce fait la dismutation ultérieure de ce corps avec l'acide pyruvique (qui résulte de la transformation de l'acide phosphoglycérique) rend également impossible la formation d'acide lactique. En fait dans la fermentation par Str. citrovorus il ne se forme pratiquement pas d'acide lactique.

4. Enfin le métabolisme de l'acide pyruvique doit ici rester le même que dans les autres fermentations : décarboxylation en acétaldéhyde. Celle-ci n'a pas de possibilité ici de se réduire, e'est-àdire de jouer le rôle d'accepteur d'hydrogène en même temps qu'elle oxyderait un triose (comme dans la fermentation alcoolique) puisque cette oxydation est déjà réalisée par l'acide hydroxyisobutyrique. Au reste, elle subit un sort différent et se transforme suivant la fin du schéma de VAN BEYNUM indiqué plus haut, c'est-àdire : formation d'acide acétique et d'alcool éthylique selon CANNIZZARo, ou condensation en aldol et isomérisation en acétoïne, On retrouve là, en milieu acide, la formation d'acétoîne et éventuellement, en oxybiose, de diacétyle. Mais ces substances ont ici leur origine dans les sucres.

5. Enfin il peut arriver que l'acétaldéhyde ne subisse aucune modification car, ainsi que l'a montré VIRTANEN, on en rencontre souvent dans les cultures aromatiques (jusqu'à 80 mgr. par litre).

Le schéma d'ensemble serait, selon nous, le suivant : 
Formation d'acétoine à rartir des sucres en présence d'acide citrique (Cas du Str. Cirrovorus)

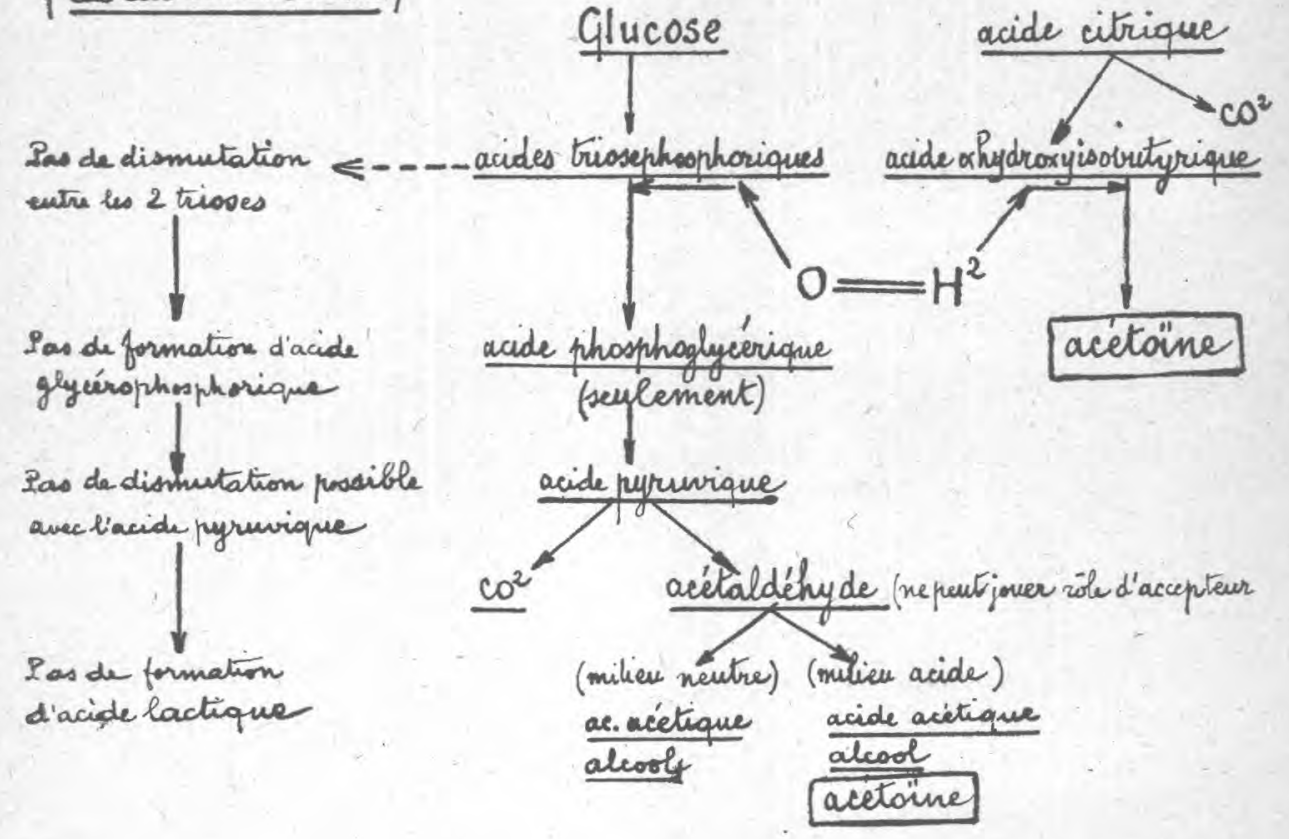

\section{En résumé :}

L'acétoïne (et le diacétyle en oxybiose) a pour origine le glucose (issu du lactose) ou l'acide citrique, suivant les cas.

Dans le cas des streptocoques lactiques aromatiques, l'acide citrique est inutile et les schémas exposés plus haut rendent compte de la formation simultanée de l'acide lactique et des substances aromatiques - celles-ci étant issues exclusivement des sucres.

Dans le cas des bétacoques, la présence de l'acide eitrique est indispensable comme accepteur d'hydrogène extérieur dans le mécanisme de la fermentation. Mais il constitue de ce fait une source d'acétoïne, par lui-même, à côté de la source normale que sont les sucres comme dans le cas précédent. Le schéma que nous proposons ci-dessus devrait donc, selon nous, remplacer le schéma initial de VAN Beynum.

\section{BIBLIOGRAPHIE}

[1] Orla-Jensen, Dairy Bacteriology (Translation P.S. Arup). Edit. Churchill, London.

[2] Schmalfuss et Barthineyer. Ztschr. Physiol. Chem., $176: 282,1928$, et Biochem. Ztschr., 216 : 330, 1929. 
[3] Van Niel, Kluyver et Derx. Biochem., Ztschr. $210: 234,1929$.

[4] Воекноut et Oтt De VRies. Zentralblatt f. Baktriol., II, 49: 373, 1919.

[5] Хтовсн. 102de Beretning fra Forsogslab., 1919.

[6] Hammer. Iowa Agr. Exp. Sta. Res. Bul., no 55, $1919 ; \mathrm{n}^{\circ \mathrm{s}} 63$ et 65, 1920 ; $n^{\circ} 67,1921$.

[7] Orla-Jensen. The lactic acid Bacteria. Publication of the Danish Academy of Sciences, 1919.

[8] Orla-Jensen. Dairy Bacteriology.

[9] Orla-Jensen. Le Lait, mars 1926, p. 161.

[10] Söncke KnUdsen. Biochem. Zeitschr., 210, 1919.

[11] Hucker et Pederson. Zentralbl. Bakt. II, Abt., 85: 65, 1931.

[12] VAn Beynum. Verslagen van Landbouwkundige Onderzoekingen, $\mathrm{n}^{\circ} 44$, 3, C, 207, 1938.

[13] Virtanen, Kontio et Storgards. Biochem. Zeitsch. $307: 215,1941$ et Angewandte Chemie, no 47-48, 491, 1941.

[14] Davies. Rapport au XI $I^{\mathrm{e}}$ Congrès mondial de Laiterie, Berlin, 1937.

[15] Vas et Csiszar. Rapport au XI Congrès mondial de Laiterie, Berlin, 1937.

[16] VAN Beynum. Rapport au XI Congrès mondial de Laiterie, Berlin, 1937.

[17] Hammer, Ia. Agr. Exp. Sta. Res. Bul., n ${ }^{\circ} 63: 59,1920$.

[18] Rumments. Rapport au XI $I^{\mathrm{e}}$ Congrès mondial de Laiterie, Berlin, 1937.

[19] Michaelian, Farmer et Hammer. Ia. Agr. Exp. Sta. Res. Bul., no 155 : $323,1933$.

[20] Hammer. Ia. Agr. Exp. Sta. Res. Bul., n 191, 1935.

[21] Stahly et Coll. Journal of Dairy Science, 1935, p. 473.

[22] Slatter. National Butter and Cheese Journal, octobre 1936, p. 20.

[23] Virtanen. Rapport au XI Congrès mondial de Laiterie, Berlin, 1937.

[24] Монг. Rapport au XI Congrès mondial de Laiterie, Berlin, 1937.

[25] Sohmalfuss et Barthmeyer. Biochem. Ztschr., $216: 330,1929$.

[26] Lemoigne. Thèse de Doctorat, Paris, 1913.

[27] Davis et Coll. Nature, 143: 558, 1939.

[28] Van Beynum. Verslagen van Landbouwkundige Onderzoekingen, $\mathrm{n}^{\circ} 42$, 11, C, 361, 1936.

[29] Matuszewski et Coll. Polish Agr. a. Forest Annual, 36, 1, 1936.

[30] Orla-Jensen. Zentrbl. Bakter, II, $86: 6,1932$.

[31] Ritter. Schweizerische Milchzeit., t. LX, 1934, p. 347.

[32] Gilurs. Communication personnelle.

[33] Hammer. Report on Agricultural Research., Ames, Iowa, 1935, p. 96. 